\title{
Hierarchies of Sensing and Control in Visually Guided Agents
}

\author{
Jana Košecká \\ Department of Computer Science, George Mason University \\ Fairfax, VA 22030-4444 \\ kosecka@cs.gmu.edu \\ http://www.cs.gmu.edu/kosecka
}

\begin{abstract}
The capability of perceiving the environment is crucial for advancing the level of autonomy and sophistication of (semi)autonomous robotic systems and determines the complexity of the tasks robotics agents can achieve. This article reviews some techniques as well as challenges shared by many applications which use visual sensing to guide the action of the robotic agent and require coordination between multiple agents. In order to support hierarchical view of such systems sensing both in the context of low-level control as well as planning and coordination between multiple mobile agents will be considered. Several examples of the design and analysis of these hierarchical hybrid systems will be outlined in the context of Intelligent Highways, namely autonomous driving and coordination between multiple vehicle and mobile robot navigation in indoors man made environments.
\end{abstract}

\section{Introduction}

Current new trends in sensing and communication give rise to many applications where computers and computer controlled devices interact with dynamically changing environment. One can encounter complex (semi)autonomous systems in a variety of domains ranging from robot exploration, teleoperation, manufacturing, automated highway systems, air-traffic management systems and coordinated underwater vehicles. The sophistication of the tasks and increase in the autonomy depends to a large extent on the capability of perceiving the environment the agent resides in. The increasing complexity of these systems brings needs for new techniques for their design and analysis as well as utilization of more complex sensors and sensing strategies. Visual sensing is making strides in the domain of embedded systems, partially due the advances in technology and speed of computing. It is now possible to use the vision as a sensor in the control loop of systems operating in unknown and unstructured environments. While the choice of elementary strategies differs across the applications, the presence of multiple sensing and control hierarchies is common to many of these types of systems.

This paper reviews the design and analysis issues of hierarchical hybrid systems and the role of visual sensing in two applications: automated highway 
systems and mobile robot domain. In automated highway applications, the elementary control strategies include automated steering, maintaining safe distance in front of the car ahead, lane change and obstacle detection. These strategies are utilized in the design of inter-vehicle coordination maneuvers so as to assure efficient highway utilization and operation at maximum capacity. In the mobile robot domain visual servoing tasks facilitate relative positioning in indoors man made environment. We also demonstrate how the composition of these elementary strategies enables landmark-based navigation, path following and obstacle detection.

From the control standpoint both of these applications can be viewed as hierarchical hybrid systems. In both cases the low-level strategies will be described in terms of of differential/difference equation and their analysis and design carried out using familiar tools and techniques from Control Theory. For the composition and coordination of these elementary strategies language of finite state machines is chosen. Finite state machine models capture the discrete event aspects of system's behavior and are used for the specification of coordination maneuvers between vehicles in automated highway applications and various goal reaching behaviors in the mobile robot domain. The language enables the specification and composition of elementary strategies, their verification and synthesis of discrete event controller of system behavior.

The presentation of the topics will be organized around two different applications. In the automated highway domain the emphasis will be on dynamic models. Vision-based controller for lateral, longitudinal control and lane change maneuver will be briefly described, followed by examples of inter-vehicle coordination maneuvers. In the domain of mobile robots, where kinematic models are appropriate, we will outline examples of visual servoing techniques for relative positioning tasks and formulate global navigation task as a sequence of relative positioning strategies.

\section{Automated Vehicles Control and Coordination}

The concept of automated highway arose as a potential solution to the current transportation problems. The main objective of the research carried out as a part California PATH project was to propose a design which would increase safety and throughput of highways by full or partial automation [15]. The automation was suggested both at the level of individual control strategies for automated driving as well as monitoring and coordination of the vehicles. The next section reviews the basic components of the AHS (Automated Highway System) architecture as well as challenges of the overall design.

\subsection{Dynamic Vision-Based Control}

The employment of the visual sensing in the context of driving is motivated by superior performance of the human visual system to accomplish these types of tasks. It is the availability of the information at the look-ahead distance, 
which makes the makes the vision sensor favorable choice for driving tasks. The dynamic behavior of the overall system rests on characterizing the dynamic model of the vehicle and the dynamics of the image features. The longitudinal and lateral dynamics of the car can be under some simplifying assumptions decoupled. The respective parts are then used for the controller design. Lateral vehicle dynamics is used for controller design. The linearized model of the vehicle retains only lateral and yaw dynamics, and is parameterized by the current longitudinal velocity. Choosing $\dot{\psi}$ and $v_{y}$ as state variables the lateral dynamics of the vehicle have the following form:

$$
\left[\begin{array}{c}
\dot{v}_{y} \\
\ddot{\psi}
\end{array}\right]=\left[\begin{array}{cc}
-\frac{c_{f}+c_{r}}{m v_{x}} & \frac{c_{r} l_{r}-c_{f} l_{f}}{m v_{x}}-v_{x} \\
\frac{-l_{f} c_{f}+l_{r} c_{r}}{I_{\psi} v_{x}} & -\frac{l_{f}{ }^{2} c_{f}+l_{r}{ }^{2} c_{r}}{I_{\psi} v_{x}}
\end{array}\right]\left[\begin{array}{l}
v_{y} \\
\dot{\psi}
\end{array}\right]+\left[\begin{array}{c}
\frac{c_{f}}{m} \\
\frac{l_{f} c_{f}}{I_{\psi}}
\end{array}\right] \delta_{f}
$$
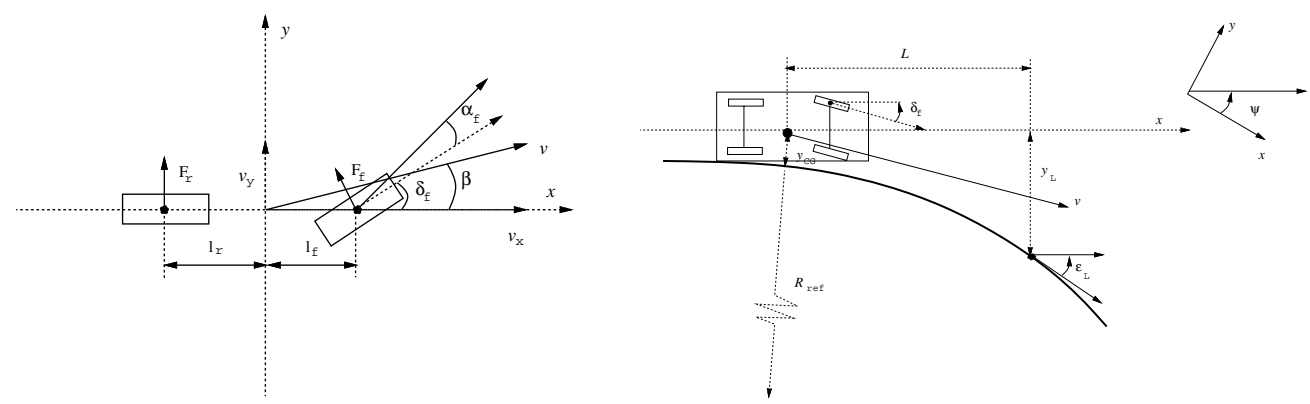

Fig. 1: The vision system estimates the offset from the centerline $y_{L}$ and the angle between the road tangent and heading of the vehicle $\varepsilon_{L}$ at some lookahead distance $L$. The motion of the vehicle is characterized by its velocity $\boldsymbol{v}=\left(v_{x}, v_{y}\right)$ expressed in the vehicle's inertial frame of reference and its yaw rate $\dot{\psi}$. The forces acting on the front and rear wheels are $F_{f}$ and $F_{r}$, respectively, $\delta_{f}$ is the front wheel steering angle, $\alpha_{f}, \alpha_{r}$ side slip angles of the front and rear tires, $\delta$ commanded steering angle, $m$ total mass of the vehicle, $I_{\psi}$ total inertia vehicle around center of gravity $(\mathrm{CG}), l_{f}, l_{r}$ distance of the front and rear axles from the $\mathrm{CG}, l$ distance between the front and the rear axle $l_{f}+l_{r}, c_{f}, c_{r}$ cornering stiffness of the front and rear tires.

The additional measurements provided by the vision system (see Figure 2.1) are: $y_{L}$ the offset from the centerline at the lookahead and $\varepsilon_{L}$ the angle between the tangent to the road and the vehicle orientation. Where $L$ denotes the lookahead distance of the vision system as shown in Figure 2.1. The equations capturing the evolution of these measurements due to the motion of the car and changes in the road geometry are:

$$
\begin{aligned}
& \dot{y}_{L}=v_{x} \varepsilon_{L}-v_{y}-\dot{\psi} L \\
& \dot{\varepsilon}_{L}=v_{x} K_{L}-\dot{\psi}
\end{aligned}
$$




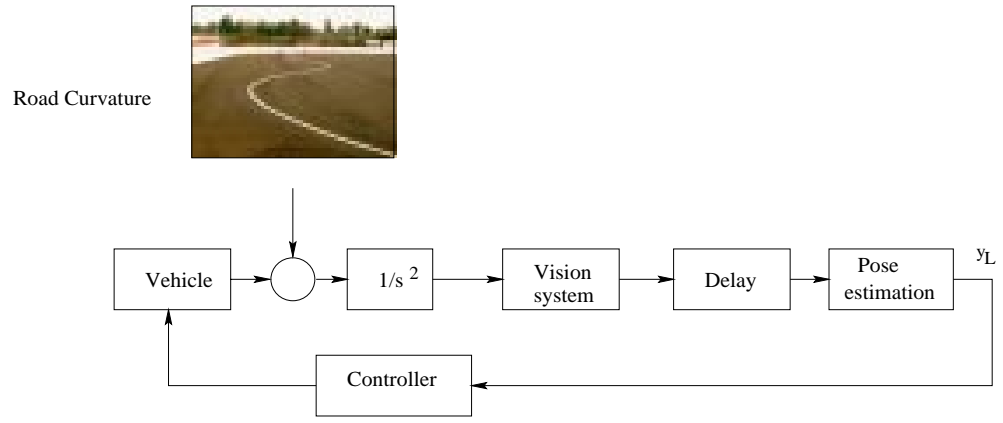

Fig. 2: Block Diagram of the lateral control strategy. Road curvature which is provided as a reference input is estimated by the vision system.

where $K_{L}$ represents the curvature of the road. The combined model with state vector $\boldsymbol{x}=\left[v_{y}, \dot{\psi}, y_{L}, \varepsilon_{L}\right]^{T}$, output $\mathbf{y}=\left[\dot{\psi}, y_{L}, \varepsilon_{L}\right]^{T}$ and control input $\boldsymbol{u}=\delta_{f}$. The road curvature $K_{L}$ enters the model as an exogenous disturbance signal $\boldsymbol{w}$. The offset at the lookahead $y_{L}$ which provides our error measurement. It is obtained by analysis of the image data acquired by a forward looking CCD video camera is mounted inside the passenger compartment. Modeling explicitly the geometric transformation between the image plane and road surface and lane marker detection, $y_{L}$ can be computed directly from image measurements. This information can be obtained at rate 30 frames per second (lane markers tracking) with a latency of 57 milliseconds $[5,8]$. Next section outlines briefly the design
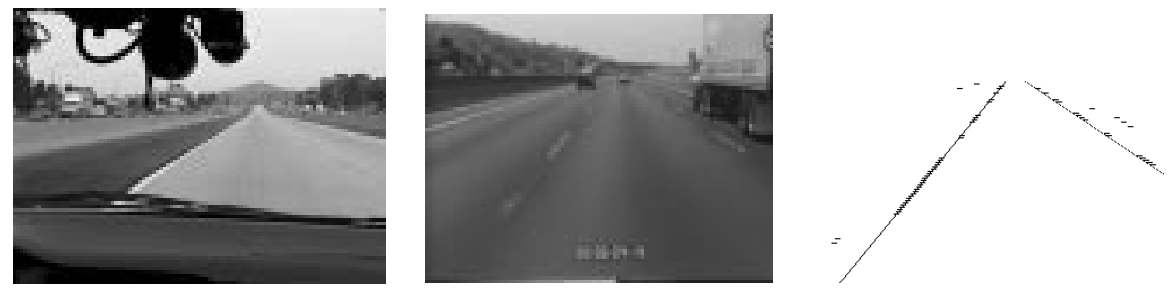

Fig. 3: These figures show the view showing the mounted cameras and performance of the lane extraction system on a typical input image.

issues and analysis of the basic control strategies.

\subsection{Control}

Lateral Control The design objective of the lateral control is to compute the appropriate steering angle, so as to maintain the desired distance of the vehicle from the center lane. The performance specifications to be achieved are close to human driving and can be characterized in terms of desirable tracking error 
$(0.1 \mathrm{~m})$, maximum allowable error $(0.4 \mathrm{~m})$, limited lateral acceleration $(0.2 \mathrm{~g})$ and sufficient passenger comfort. Furthermore successful control design should be also robust with respect to the variety of road conditions and car parameters (mass, friction, cornering stiffness etc). Using standard analysis techniques in the frequency domain, the stability of the lateral control can be achieved over the entire range of operating speeds. This is possible even in the presence of the delay which is explicitly modeled. The delay can be compensated by the additional phase lead provided by increasing the look-ahead distance. In addition to measurements are obtained from the on-board fiber optic gyro it is possible to design an observer capable of estimating the curvature of the road $K_{L}$. This information is used for anticipatory control as a part of the vehicle feedforward control law. Detailed description of the control law can be found in [8].

Lane Change Maneuvers Lane change maneuvers are accomplished by supplying a reference trajectory, $y_{L}(t)$, as an input to the lateral control systems. This reference trajectory is a simple fifth order spline which smoothly moves the vehicle from one lane to another. The curvature of the reference trajectory is also supplied as an additive input to the feedforward control law. The actual controller is then identical to the lateral control strategy, which is given this virtual reference trajectory.

Longitudinal vehicle control For the purpose of brake and throttle control for keeping the safe distance in front of the car ahead, both laser radar and vision sensing is used for measuring the distance of the car ahead. The visual sensing in this case combines shape reconstruction (2D planar reconstruction rather than the usual 3D) from stereo/motion with motion estimation, using robust and efficient feature matching methods. The resulting algorithm runs at $3-5 \mathrm{~Hz}$, and frame-rate performance $(30 \mathrm{~Hz})$ and performs well over large periods of time.

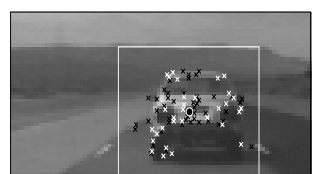

1

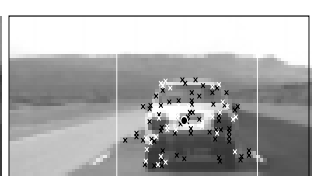

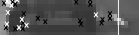

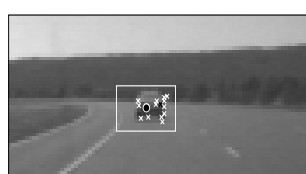

$421 \ldots$

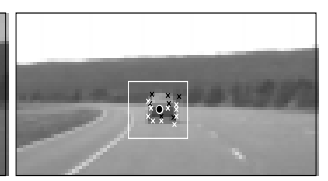

Fig. 4: Example stereo-pairs from the tracking sequence. The corner features are shown as small crosses, white for those matched over time or in stereo, and black for unmatched features. The black and white circle indicates the position of the fixation point, which ideally should remain at the same point on the lead car throughout the sequence. The white rectangle describes the latest estimate of the bounding box for the vehicle. 
These elementary strategies constitute the lowest level of control and sensing hierarchy. The standard control theory techniques guide their design and enable their analysis. Presented are examples of vehicle runs at approximately $75 \mathrm{mph}$, along 7 mile curved oval. In the absence of feedforward control law, the vehicle has some steady state error in the curved section of the track. Figure 5 describes the performance of tested control strategies without and with the feedforward term.

a.
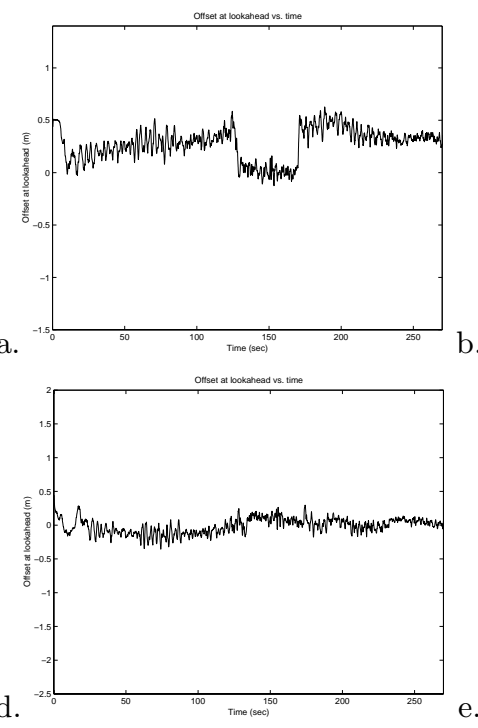
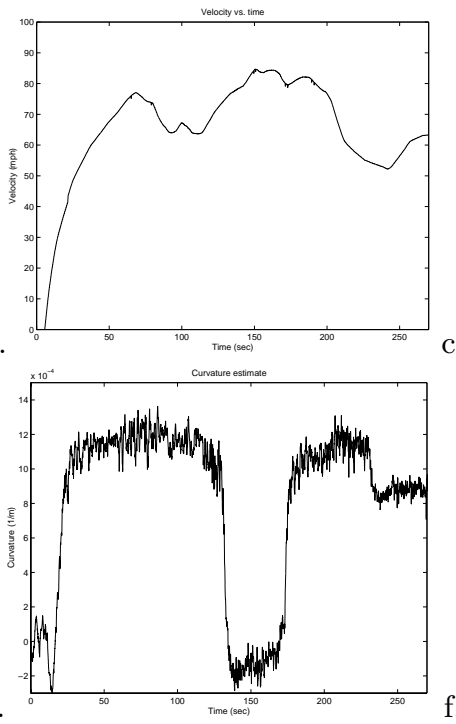

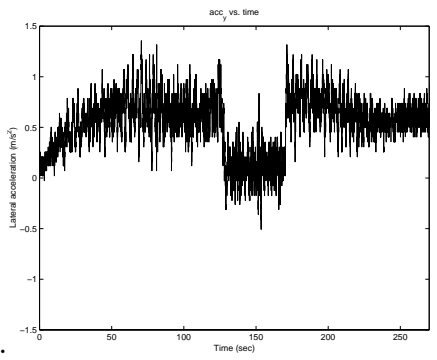

acc, vs. time

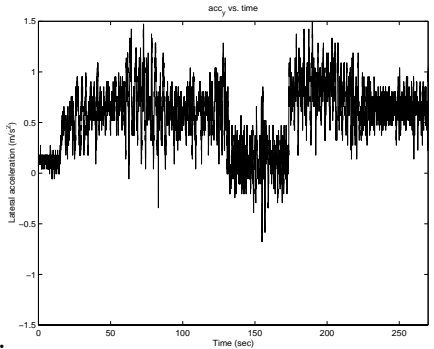

Fig. 5: This figure depicts the performance of the lead-lad controller. Figures $\mathrm{a}, \mathrm{b}$ and $\mathrm{c}$ indicate the offset of the centerline of the road at a distance of 15 meters ahead of the vehicle, velocity profiles used in the runs and the lateral acceleration experienced at the center of gravity of the vehicle. Figures d, e and f demonstrate the effect of the feedforward control term on the overall tracking performance, in particular the offset at the lookahead, the curvature estimate used in the feedforward term and the last row shows the lateral acceleration profile. Notice that the steady state offset in the curved sections was essentially eliminated.

\subsection{Vehicle Coordination}

The main goal of coordination of multiple vehicles in AHS is to reduce congestion and maximize highway throughput. Towards this end a platooning concept has been explored [15]. The main idea behind the concept is to organize vehicles into closely spaced platoons, where the vehicles travel at high speeds at close distances with respect to each other. The vehicle can operate in two different 
modes: the follower mode and the leader mode. The switching between the modes is achieved by means of five maneuvers: join, split, lane change, entry and exit, as demonstrated in Figure 6. Additional lane number information is a part of the mode.

a.

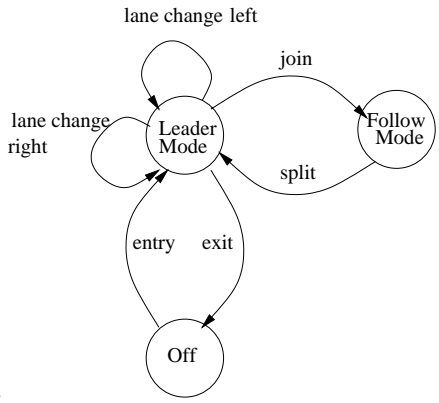

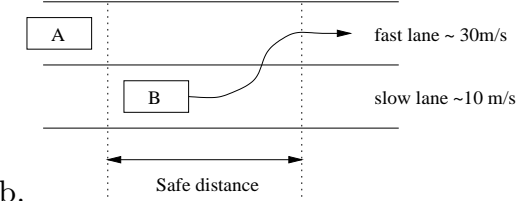

b.

Fig. 6: a. The transitions between different vehicle models, indicate different maneuvers with separately designed communication protocols. b. An example of an unsafe lane change scenario.

The individual maneuvers have associated communication protocols which have been designed in [4]. The coordination protocols exchange information between individual vehicles. When the conditions for the maneuver execution are met, the regulation level executes the appropriate maneuver. For the purpose of discrete verification, the behavior of the continuous controller has been abstracted to the level of finite state machines, which capture the discrete behavior of the sensors and controllers. This enables verification of the logical correctness of the protocols using verification tool COSPAN [3]. In spite of the correctness of the analysis at continuous and discrete event levels of the system, the overall simulation revealed crashes [2].

This indicated that certain aspects of systems behavior were not captured by models at individual levels and proved to be crucial for being able to guarantee safe design. An example of the lane change maneuver of a single vehicle was one such an instance. The basic logic of the lane change maneuver protocol first checked whether there is sufficient space in the lane ahead, and provided that the free space would guarantee the safe distance from the neighboring vehicle the maneuver was executed. In case there was no other vehicle in the next lane in sight the maneuver was immediately executed. In the initial state as depicted in Figure 6 (right), the lane change from the slow to fast late has been enabled. However due to the differences in lane speeds the vehicle was behind was unable to deaccelerate as to prevent the collision with the vehicle ahead. The main cause of this unsafe operation were physical limits of sensors and acceleration and deacceleration limits of the individual vehicles. None of these aspects was captured by the respective levels of hierarchical control system which have been 
analyzed. This and several other instances of such examples were documented in $[2]$.

Based on these observations alternative techniques for proving the safety of the overall hybrid system design for these particular scenarios have been proposed. In [10] authors advocate the game theoretic approach, where the correctness of the overall hybrid controller can be proved by design, with no need for subsequent verification. Assuming that the designer can determine the control input, all other inputs are considered as disturbance (either discrete or continuous). Given this setting a set of safe initial conditions can be calculated. If the initial conditions of the system are in the safe set the maneuver can be executed, regardless of the disturbances. The advent of this approach has been further explored in the context of other maneuvers as well as other hybrid control problems [14].

\section{Mobile Robot Navigation}

While the tools and techniques for the analysis of hierarchical hybrid systems developed in the context of AHS are general, the mobile robot domain raises some additional issues and differences. Richness of the future mobile robot environments makes the sensing problem more challenging. This in turn also increases the complexity, level of autonomy as well as variety of robotic tasks. Next section reviews some of the sensing and hierarchical control issues in the context of vision based mobile robot navigation.

\subsection{Visual Servoing}

In the mobile robot domain we will introduce visual servoing approach which enables formulation of the control objectives using the image measurements directly in the image plane. The overall approach consist of three steps: defining a set of features in the image, specifying their desired goal configuration, and applying a control law that ensures the convergence from any initial configuration to a desired goal configuration in the image. The approach has been originally introduced in [1]. Suppose in general terms that the camera system is considered as a dynamical system $\dot{g}=f(g, u)$ where $u=(\omega, v)$ is the angular and linear velocity $\omega, v \in \mathbb{R}^{3}$ of the camera system and $g \in S E(3)$ is the pose of the camera. The image feature is typically defined implicitly as a function of pose $h(g, \mathbf{x})=0$. Then given some desired position of the image features, the robot task can be formulated as a regulation of a cost function $J\left(\mathbf{x}, \mathbf{x}_{d}\right)$ during the time interval $[0, T]$. Most commonly used control law is a simple gradient based scheme, which is typically sufficient for exponential convergence of the output to zero:

$$
u=\arg \min _{u} \dot{\mathbf{x}}^{T} \nabla J
$$

Convergence and stability can be assessed using standard control theory techniques. In the next section we will formulate several visual serving tasks using 
kinematic models. In the mobile robot domain the speeds are relatively low and dynamic effects are not so prevalent so the use of kinematic models is well justified.

Mobile Robot Kinematics The kinematic model of the mobile robot used in our experiments is that of a unicycle. Let $p_{f m}(t)=(x, y, z)^{T} \in \mathbb{R}^{3}$ be the position vector of the origin of frame $F_{m}$ from the origin of frame $F_{f}$ and the rotation angle $\theta$ is defined in the counter-clockwise sense about the $y$-axis, as shown in Figure 7 . For the unicycle kinematics, $\theta(t)$ and $p_{f m}(t)$ satisfy:

$$
\begin{aligned}
& \dot{x}=v \sin \theta \\
& \dot{z}=v \cos \theta \\
& \dot{\theta}=\omega
\end{aligned}
$$

where the steering input $\omega$ controls the angular velocity $\dot{\theta}$; the driving input $v$ controls the linear velocity along the direction of the wheel. Monocular camera mounted on the mobile robot is facing downward with a tilt angle $\phi>0$ and the camera is elevated above the ground plane by distance $d$, as shown in Figure 7 . Then the kinematics of a point $q_{c}=(x, y, z)^{T}$ attached to the camera frame $F_{c}$
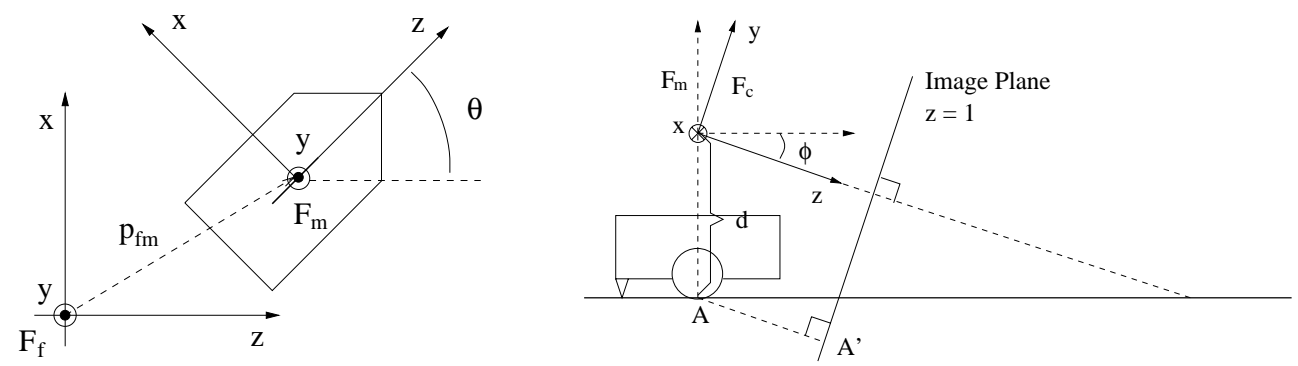

Fig. 7: Model of the unicycle mobile robot and the side-view of the unicycle mobile robot with a camera facing downward with a tilt angle $\phi>0$.

is given in the (instantaneous) camera frame by:

$$
\left(\begin{array}{c}
\dot{x} \\
\dot{y} \\
\dot{z}
\end{array}\right)=\left(\begin{array}{c}
0 \\
\sin \phi \\
\cos \phi
\end{array}\right) v+\left(\begin{array}{c}
y \sin \phi+z \cos \phi \\
-x \sin \phi \\
-x \cos \phi
\end{array}\right) \omega .
$$

An instance of the general visual servoing problem considered here is that of following arbitrarily shaped curve. Detailed treatment of this scenario can be found in [12]. Consider planar ground curve $\Gamma$ which can be expressed in the camera coordinate system as $\Gamma=\left(\gamma_{x}(y, t), y, \gamma_{z}(y)\right)^{T}$. Since $\gamma_{z}(y)$ does not change as a function of time, the dynamics of the ground curve can be expressed using (5) as:

$$
\dot{\gamma}_{x}(y, t)=-\left(y \sin \phi+\gamma_{z} \cos \phi\right) \omega .
$$


The shape of the orthographic projection ${ }^{1}$ of the ground curve $\tilde{\Gamma}=\left(\gamma_{x}(y, t), y\right)^{T}$ then evolves in the image plane $z=1$ according to the following Riccati-type partial differential equation.

$$
\frac{\partial \gamma_{x}}{\partial t}=-\left(y \sin \phi+\gamma_{z} \cos \phi\right) \omega+\frac{\partial \gamma_{x}}{\partial y}\left(v \sin \phi-\gamma_{x} \omega \sin \phi\right) .
$$

The above expression captures the dynamics of the ground plane curve. We can

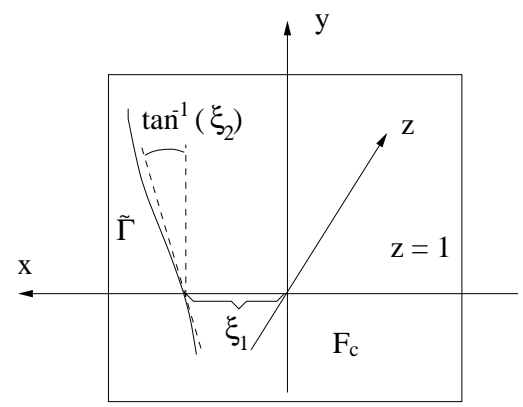

Fig. 8: The orthographic projection of a ground curve on the $z=1$ plane. Here $\xi_{1}=\gamma_{x}$ and $\xi_{2}=\frac{\partial \gamma_{x}}{\partial y}$.

the consider a feature function $F=x-\xi_{1}(y, t)$, which can be directly measured in the image and which we will try to regulate, so as to follow the curve. The relationship between $\xi$ and $\gamma$ is expressed in caption of Figure 8 in case of orthographic projection. The dynamics of the image curve can be expressed concisely in terms of two vector fields $f_{1}$ and $f_{2}$ and behaves as a nonlinear system of the form:

$$
\dot{\xi}=f_{1}(\xi) \omega+f_{2}(\xi) v
$$

It can be shown that if the given ground curve is a linear curvature curve, the above system is completely controllable and observable and can be globally exponentially tracked given the following control law:

$$
\begin{aligned}
v & =v_{0}+\xi_{1} \omega, \quad v_{0}>0 \\
\omega & =\frac{\sin \phi}{1+\sin ^{2} \phi \xi_{2}^{2}}\left(v_{0} \sin \phi \xi_{3}+a \xi_{1}+b \xi_{2}\right), \quad a, b>0 .
\end{aligned}
$$

Then the partial closed loop system of $\xi_{1}, \xi_{2}$ is linearized and given by:

$$
\begin{aligned}
& \dot{\xi}_{1}=v_{0} \sin \phi \xi_{2} \\
& \dot{\xi}_{2}=-a \xi_{1}-b \xi_{2}
\end{aligned}
$$

Discussion on control inputs for the general steering problem from one point to another can be found in [12].

${ }^{1}$ From the control point of view the perspective and orthographic projection models can be treated as equivalent in this problem. 
Wall following A variation of this general strategy can be then used for formulating the wall following as a special case of following a zero curvature curves. The original control law can be used for successful tracking of corridors, while connecting them by virtual circular segments. An example of an image pair of initial and steady state as seen by the camera is shown in Figure 9. The control
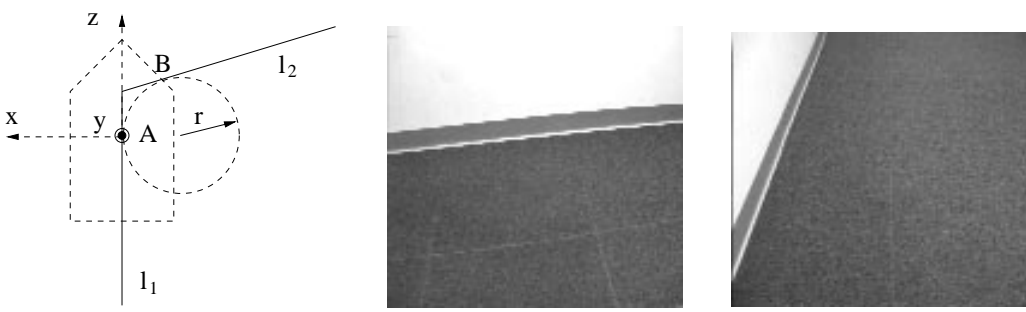

Fig. 9: Piecewise constant curvature curves for wall following. Initial and desired view of the straight line feature. The line corresponds to the intersection of the ground plane and vertical wall plane.

strategy for following arbitrary curves, can be also used for path following, where the curve will be a virtual one, computed by a path planner.

Door Servoing Another relative positioning strategy useful for navigation task is the door servoing strategy. For this control problem we employ an additional degree of freedom: the pan angle $\psi$ of the camera mounted on the turntable. This allows search for the doorway over an extended field of view. The objective of the door servoing task is to position the mobile robot with respect to the doorway so it can pass through it. The control needs to take into account the nonholonomic constraints of the vehicle in slightly different way. The doorway is defined by two points at the same height above the ground plane located at the door frame. An example views of a doorway from an initial and desired configuration is shown in Figure 10. In this scenario the control of the mobile base and the control of the
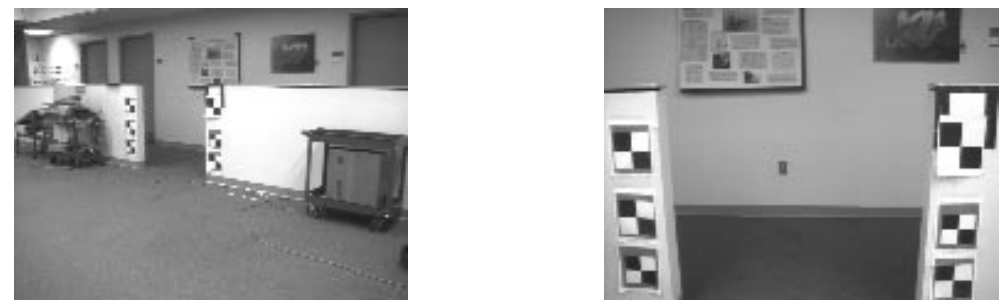

Fig. 10: Initial and desired view of the doorway features.

pan platform are decoupled. Few solutions to this regulation problem has been 
proposed in [7]. In terms of visual sensing the difficult part of the employment of these strategies in dynamically changing environments is initialization and tracking in the presence of multiple moving objects.

\subsection{Composition of elementary strategies}

Previous section outlined some sensing and control strategies in the mobile robot domain. Together with point-to-point navigation and obstacle avoidance described in [9], different navigation tasks can be formulated. In the presence of manipulators as well as additional sensors (e.g. ultrasound), different lowlevel controllers and sensing routines can be developed, which constitute basic capabilities of the mobile robot. Combination of these elementary strategies en-

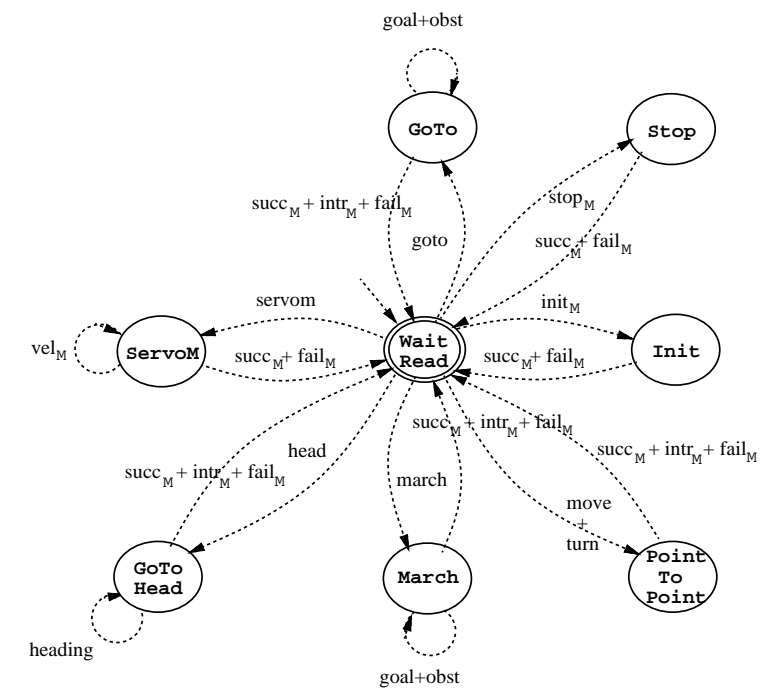

GoTo

$$
\begin{aligned}
& \Sigma_{\mathrm{C}}=\left\{\text { move, turn, } \text { servo }_{\mathrm{M}}\right. \text {, goto, head, march, init } \\
& \mathrm{M} \\
& \left.\Sigma_{\mathrm{U}} \text {, stop }\right\}
\end{aligned}
$$

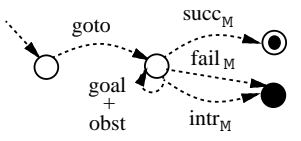

Fig. 11: a. Mobile base server process and it's modes. b. Discrete event abstraction of the elementary control strategy for goal directed navigation.

ables accomplishment of bigger variety of tasks. This brings out new needs for specification and composition of the elementary strategies. Similarly as in the intelligent highway scenarios, the discrete event abstractions of the basic control modes need to be established. An example of such model for the mobile robot base is in Figure 3.2. In addition to the discrete event model of the controlled system (mobile base in this case), discrete event abstractions of the elementary control strategies capture the discrete aspects of continuous behavior such as initialization, failure, change of set point, as depicted in Figure 3.2. The details of 
the process can be found in [6]. Similar, robot based schema approach, has been proposed by [11]. Adopting a process view of the elementary control strategies, set of composition operators is introduced, whose semantics is defined in terms finite state machines. The basic composition operators comprise are:

Sequential composition $P=R ; S$. Process $P$ behaves like $R$ until $R$ terminates and then behaves like $S$. $P$ terminates when $S$ terminates and has the same termination status as $S$.

Parallel composition $P=R \| S$. Process $P$ behaves like $R$ and $S$ running in parallel. $P$ terminates with the termination and status of the last terminated process ${ }^{2}$.

Conditional composition $P=R\langle v\rangle: S(v)$. Process $P$ behaves like $R$ until $R$ terminates successfully computing $v$ which is then used to initialize process $S^{3}$ If $R$ fails the composition fails.

Disabling composition $P=R \sharp S$. Disabling composition is similar to parallel but if one of the processes terminates the other process is terminated as well. $P$ has the same termination status as the process that caused the termination of the composition (i.e., the process that finished first).

Synchronous recurrent composition $P=R\langle v\rangle: ; S(v)$ is recursively defined as $R<v>: ; S(v)=R<v>:(S(v) ;(R<v>: ; S(v)))$. This composition terminates with the failure of process $R$.

Asynchronous recurrent composition $P=R<v>:: S(v)$ is recursively defined as $R<v>: ; S(v)=R<v>:(S(v) \|(R<v>:: S(v)))$. This composition terminates with the failure of process $R$.

Given the composition operators more complex tasks can be expressed in terms of composition of elementary strategies. Such description can be then viewed as specification of a task. Given specification can be automatically translated to the finite state machine representation and using techniques proposed by [13], discrete event supervisor can be synthesized. In addition to the task additional properties of the system can be verified.

In the mobile robot domain the dynamic effects are not so prevalent as in AHS domain, however due to bigger variety and complexity of the tasks it is of importance in some domains that certain notion of task accomplishment can be guaranteed. This rests on global assumptions the sensing strategies make about the environment. Some of these issues will be now demonstrated in the context of global navigation tasks.

Global Navigation Given a set of relative positioning strategies, the global navigation task can be accomplished as a sequence of relative positioning tasks [7]. That sequence is determined based on topological representation, which is assumed to be given. This topological abstraction is determined by visibility regions associated with individual landmarks and is captured by a place graph. A corresponding place graph given a set of landmarks is depicted in Figure 12. Individual nodes in the place graph represent the intersections of the visibility

\footnotetext{
${ }^{2}$ The composition of parallel processes requires synchronization of the processes in terms of shared events. The exact formulation of this is described in [6].

3 The variable $v$ is a global variable. We assume shared memory model, which allows the access to a global variable, that may be accessed by multiple processes.
} 

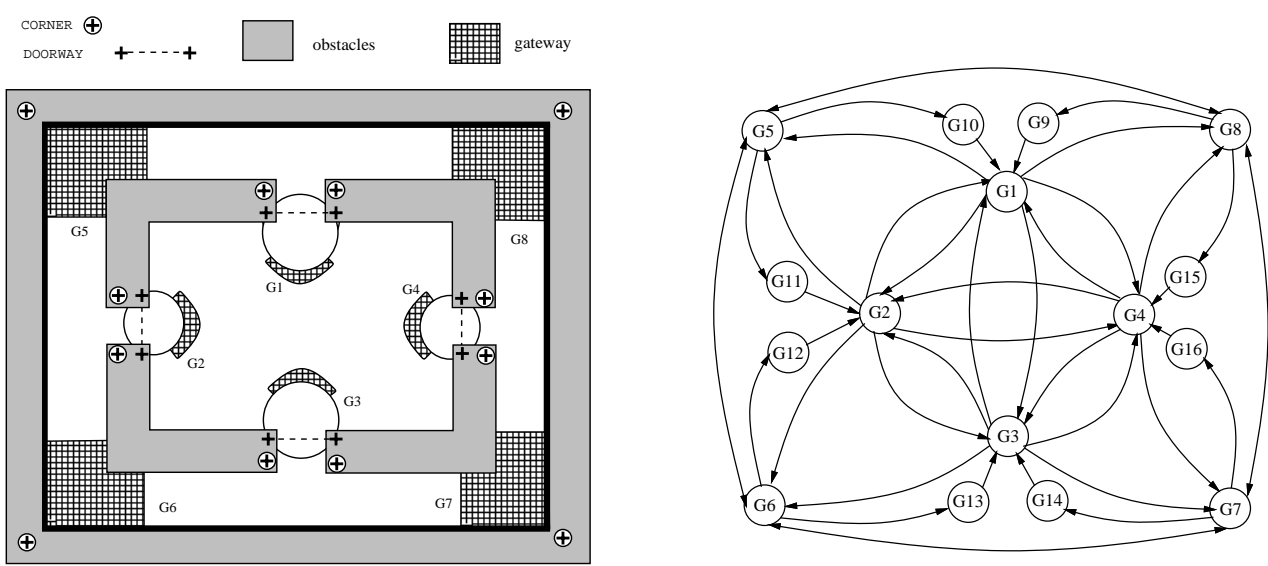

Fig. 12: The gateway regions correspond to the nodes of the place graph. Some of the gateways were omitted for clarity. Place graph corresponding to a set of landmarks chosen in our laboratory. $G 2$ corresponds to the gateway between the door and the boundary of the wall leading to two corner landmarks. The edge between $G 2$ and $G 5$ is labeled by a wall following strategy and the executed path is Figure 8. Some of the gateway nodes and edges were omitted from the graph for clarity.

regions of specified landmarks or boundaries leading to landmarks. The edges coming out of the node correspond to the possible servoing strategies that can be applied from a given place. A node is characterized by a set of visible or approachable landmarks, i.e., set of landmarks with respect to which the mobile robot can be positioned. In the case every point in the free 2-D configuration space belongs to a certain place, the place graph is fully connected.

What follows is an example of the door servoing task expressed in task specification language introduced previously. The initialization of doorway features can be written in a following way:

$$
\text { InitServo }\left(\text { door }_{i}\right):=\operatorname{Search}(s) \| \operatorname{ServoC}
$$

where we utilize the degree of freedom of the pan platform. Once the door features are found the actual servoing task can be initiated:

$$
\text { Servo }:=\operatorname{Track}\left(s_{d}, s\right) \| \text { ServoM } \| \text { ServoC }
$$

where ServoC and ServoM are control strategies for mobile base and camera platform and Track is the feature detection and tracking routine which at each instance of time generates appropriate velocity commands to both camera pan platform and mobile base controllers. A single relative positioning task, expressed in task specification language, can be then written as:

$$
\text { RelPosition }\left(\text { door }_{i}, s_{d}\right):=\operatorname{InitServo}\left(\text { door }_{i}\right)<s>\text { : Servo }\left(s, s_{d}\right)
$$


where : is the conditional composition operator, which specifies that the first strategy must be completed successfully before the second one can be initiated. By labeling the individual arcs of the place graph by expressions of the above form, we can then generate the global plan as a result of a place graph search.

\subsection{Discussion}

The outlined approach demonstrates the use of relative positioning strategies with respect to the set of naturally occurring landmarks for global navigation. In general in the domain of mobile robots residing in structured man made environment, the main challenge lies in the proper initialization of the control strategies and acquisition, detection and instantiation of an environment model. These functionalities are central in order to be able to specify and execute more complex goal directed behavior of the robotic agent and move beyond systems, which require hand initialization and are designed specifically for a particular task. In in this article we reviewed mainly deterministic aspects of hierarchical hybrid systems. The need for probabilistic formulations is apparent when in comes to using modeling and interpretation of noisy sensory readings as well as outcomes of robot's actions.

\section{References}

1. B. Espiau, F. Chaumette, and P. Rives. A new approach to visual servoing in robotics. IEEE Transactions on Robotics and Automation, 8(3):313 - 326, June 1992.

2. D. Godbole and J. Lygeros. Hierarchical hybrid control: A case study. In Proceedings from IEEE Conference on Decision and Control, pages 1592-1597, 1994.

3. Z. Har'El and R. Kurshan. Cospan user's guide. ATEST Laboratories, 1987.

4. A. Hsu, F. Eskafi, E. Singh, and P. Varaiya. Protocol desing for an automated highway system. Discrete Event Dynamic Systems, 2(1):183-206, 1994.

5. J.Malik, P. McLauchlan C.J. Taylor, and J. Košecká. Development of binocular stereopsis for vehicle lateral control. path mou-257 final report. Technical report, Department of EECS, UC Berkeley, 1997.

6. J. Košecká and H. Ben-Abdallah. An automaton based algebra for specifying robotic agents. In Proccedings of the AMAST Real-Time Systems Workshop, Salt Lake City, Utah, 1996.

7. J. Košecká. Visually guided navigation. Robotics and Autonomous Systems, 21(1):37-51, July 1997.

8. J. Košecká, R. Blasi, C.J. Taylor, and J. Malik. Vision based lateral control of vehicles. In Conference on Intelligent Transportation Systems, Boston, 1997.

9. J. Košecká and H. I. Christensen. Experiments in behavior composition. Journal of Robotics and Autonomous Systems, 19(3/4), 1997.

10. J. Lygeros and D. Godbole. Verified hybrid controllers for automated vehicles. IEEE Transactions on Automatic Control, 43(4):522-539, 1998.

11. D. M. Lyons and A. J. Hendriks. Planning for reactive robot behavior. In Proceedings of the IEEE International Conference on Robotics and Automation, pages $2675-2680,1992$. 
12. Yi Ma, Jana Košecká, and Shankar Sastry. Vision guided navigation of a nonholonomic mobile robot. IEEE Transactions on Robotics and Automation, 15(3):521536, 1999.

13. P.J. Ramadge and W.M. Wonham. Supervisory control of a class of discrete event processes. SIAM J. Contr. Optimization, 25(1):206-230, 1987.

14. S. Sastry, G. Meyer, C. Tomlin, J. Lygeros, , D. Godbole, and G. Pappas. Hybrid control in air traffic management systems. In Proceedings of the IEEE Conference in Decision and Control, pages 1478-1483, 1995.

15. P. Varaiya. Smart cars on smart roads: problems of control. IEEE Transactions on Automatic Control, AC-38(2):195-207, 1993. 